\title{
MegaJournal: revisão de conceitos
}

\author{
Milton Shintaku \\ Instituto Brasileiro de Informação em Ciência e Tecnologiamilton. \\ shinshintaku@ibict.br \\ Ronnie Fagundes de Brito \\ Instituto Brasileiro de Informação em Ciência e Tecnologia \\ ronniefbrito@gmail.com \\ Piotr Trzesniak \\ Universidade Federal de Itajubá \\ piotreze@gmail.com
}

Como citar:

SHINTAKU, M.; BRITO, R. F de; TRZESNIAK, P. Revistas técnico científicas vinculadas ao poder executivo. In: ABEC MEETING, 2, 2018, São Paulo. Anais... São Paulo: Associação Brasileira de Editores Científicos, 2018. p. 1-6.

http://dx.doi.org/10.21452/abecmeeting.2018.157

\section{RESUMO}

O MegaRevista (MegaJournal) tem se apresentado como uma opção de modelo de revistas científicas em ambiente totalmente digital, com altas taxas de publicação e avaliação pelos pares simplificada. Entretanto, esse tipo de publicação ainda não é muito difundido no Brasil, requerendo estudos que disseminem seus conceitos e práticas. Nesse sentido, o presente estudo faz um revisão de literatura sobre o tema, por meio de pesquisa bibliográfica. Assim, os MegaRevistas datam de 2011, podendo ser uma evolução dos periódicos eletrônicos de acesso aberto, mas não se pode afirmar que estão totalmente firmados, requerendo estudos mais profundos, mesmo que suas características primárias já tenham sido estabelecidas.

Palavras-Chave: Megaperiódicos. Publicação científica. Comunicação científica.

\section{ABSTRACT}

The MegaJournal presents as an option of journal's model with high frequency of publication and simplified peer review, in a digital environment. However, this journal's model is not disseminate, in Brazil, therefore it requires studies about concepts and practices. Thus, the aims of this work is to present the theme literature, through bibliographic records. Along these lines, the MegaJournal date from 2011 and may be a evolution of the open access online journals, on the other hand it can not allege that the theme is endorse, because further research regarding the theme are necessary, even if their primary characteristics already been established. Keywords: MegaJournals. Journals. Scientific communication. 


\section{INTRODUÇÃO}

A comunicação científica passa por adequações às novas tecnologias e tendências da ciência. Nota-se que o movimentos científicos atuais como a Ciência Aberta (Open Science), Dados Abertos (Open Data) e e-Science, entre outros, tem apresentado desafios a certas práticas estabelecidas, da mesma forma em que a evolução das Tecnologias da Informação e Comunicação (TICs) têm desenvolvido novas ferramentas de apoio às atividades científicas.

Hurd (2000) prediz mudanças em práticas na comunicação científica para curto prazo, com grande uso de tecnologias, no qual toda a disseminação da informação será feita por meio digital, por meio de vários canais. O uso cada vez maior das tecnologias nos processos de disseminação do conhecimento científicos transcende as barreiras impostas pelo suporte físico, além de promover maior rapidez no processo. O impacto da tecnologia na comunicação científica é indiscutível, tanto que, Hartgerink e Van Zelst (2018) advogam pelo término da tradicional disseminação de pesquisas concluídas, sendo substituída pela disseminação dos resultados parciais, aumentando o número de artigos produzidos para uma mesma pesquisa, como já ocorre em algumas disciplinas em que as pesquisas são longas. Assim, requerendo publicações mais rápidas no processo de editorial. Este ponto pode-se alinhar aos sistemas de informação denominados Current Research Information System (CRIS), que visam divulgar as pesquisas em andamento, incluindo os seus resultados.

Nesse contexto complexo e em mutação, tem-se a presença da chamada MegaRevista, do inglês MegaJournal, que para Parcker (2012) é resultado da autonomia oferecida pelos documentos digitais, que não impõe limite aos editores, quanto a número de artigos publicados, nem limitações de volumes e números. Dessa forma, sugere-se ser a evolução das revistas científicas atuais, sem as barreiras impostas pelo suporte físico.

Entretanto, Wakeling et al (2017), levantando informações sobre MegaRevistas (MR), não encontraram evidências do conhecimento desse tipo de publicação entre pesquisadores, indicando a pouca familiaridade desse canal de disseminação científica. Nesse contexto, o objetivo deste estudo é discutir as MR, na medida em que ainda são novidade para muitos editores e pesquisadores, levantando e analisando informações desse canal de disseminação da informação, de forma a apresentar uma base conceitual, que apoie futuros estudos sobre o tema. Assim, colaborar com a discussão sobre MR no Brasil, que ainda não possui muitas publicações sobre o tema.

\section{METODOLOGIA}

O estudo possui abordagem qualitativa, utilizando técnicas de levantamento bibliográfico. Para tanto, utilizaram-se em parte as técnicas apresentadas por Treinta et al (2014), devido a quantidade de informações existentes sobre o tema, principalmente na língua inglesa. Selecionaram-se as fontes de informações para o estudo por meio dos eixos de relevância do relacionada ao tema, autor, periódico e artigo.

\section{RESULTADOS}

Em 2011, Frantsvag (2011) anunciava o surgimento das MR, como um dos tópicos tratados durante a $3^{\text {a }}$ Conferência de Publicação acadêmica em Acesso Aberto, 
no qual pública grande quantidade de artigos desde que atendam aos seus critérios, de forma contínua e rápida. Dessa forma, não segmenta o conhecimento publicado, em publicações mais amplas em termo de temática e dando vazão à demanda por publicação.

Binfield (2012), editor da Public Library Of Science (PLOS), avalia que o PLOS possui as características de uma MR, sendo possivelmente o primeiro a ser publicado. Para o editor da PLOS, o processo editorial e avaliação pelos pares efetuados de forma simplificado dá a rapidez necessária a grande quantidade de artigos publicados. Entretanto, o referido autor advoga que as MR requerem estudos mais profundos de forma a verificar formas mais eficientes de medir o impacto desses canais de publicação.

No âmbito da temática de Informação e Documentação, Lópes-Borrull (2014) afirma que uma $M R$, que integre revistas já existentes pode assemelhar-se tecnicamente a uma evolução de repositório temático, com adição do fluxo de avaliação pelos pares às funcionalidades do repositório. Esse ponto pode corroborar com a visão de repositórios ligados à Ciência Aberta (Open Science), que implementam a avaliação aberta, como relatado por Kriegeskorte (2012), no qual substitui o processo de submissão pelo autodepósito.

Para Bjork (2015), as MR deve atender às seguintes características: Grande volume de publicação por ano; Ampla cobertura temática; Publicação em Acesso Aberto; Formas diferenciadas de revisão pelos pares, podendo implementar o Article Processing Charge (APC); Altas taxas de aceitação para publicação e Rapidez do processo editorial. Já Pinfield, Willett, Wakeling (2017) adicionam as características secundárias de APC moderado; Publisher de prestígio; Reuso de dados; e Altimetria como métrica.

Numa visão mais ampla, voltada à comunicação científica, Burns (2015) afirma que os MR podem facilitar a geração de indicadores úteis à cientometria, visto que já nasceram em meio digital e são interdisciplinares. Assim, os MR podem ajudar a verificar os problemas na comunicação científica em ambiente totalmente web e com uso das novas tecnologias, como seus novos padrões e práticas, possibilitando apresentar cenários mais precisos sobre padrões publicação.

Domnina (2016) corrobora a ideia de que MR forma repositórios contendo uma grande quantidade de artigos oriundo de várias disciplinas, no qual a visibilidade é efetuada por métricas de uso individual, diferente do fator de impacto que é da revista. Dessa forma, possibilita que pesquisadores de temas distintos publiquem em uma mesma MR e retirando um pouco a influência do corpo editorial na seleção dos temas a serem publicados, além de individualizar a geração de indicadores por artigo.

Os MR têm se apresentados promissores, tanto que, Shopovsky e Marolov (2017) verificaram a aceitação dos autores em publicar em nesses canais, revelando estarem satisfeitos com o seu processo editorial. Já Lazaroiu (2017) considera os MR como opção para as publicações científicas, tanto de acesso livre, com pagamento de APC e de assinatura, na medida em que adota políticas mais flexíveis e atuais.

Björk (2018), por sua vez, revela que a MR se estabelece em um nicho próprio dentro do ecossistema da comunicação científica, atendendo a necessidade de grupo de autores que requerem rápida avaliação e publicação. Cabe destacar que o uso das tecnologias de comunicação e informação impõe certa velocidade aos processos, não sendo diferente aos processos de disseminação da informação nas ciências. 


\section{CONSIDERAÇÕES FINAIS}

As MRs são um canal inovador de publicação de artigos, pois implementam novas regras de negócio diferentes dos periódicos tradicionais. Atuam buscando aproveitar as oportunidades do ambiente web, ampliando o foco temático de publicação e promovendo o uso de novas métricas como forma de avaliação. Wakeling et al (2017) revelam crescimento das publicações de artigos na MR medicine, mas com queda no fator de impacto, visto que publicam mais artigos, diluindo as citações, mas não demonstrando perda de prestígio da revista.

Pouco estabelecido no Brasil, apresenta oportunidade para discussão e desafio, visto que podem representar um novo modelo de sustentação aos periódicos nacionais, muitos dos quais tem dificuldades em manter periodicidade e fluxo de publicação, assim com seu corpo editorial e de avaliadores. As associações científicas e universidades formando consórcios para manter o MR, juntando esforços para melhorar a disseminação dos resultados de pesquisas brasileiras.

Pode-se afirmar que as MR ainda estão se estabelecendo e mudando algumas regras de publicação científica, mas como defendem Spezi el al (2017), as MR não são publicações que se homogenizam nas características, combinam-as de forma a cada um ter identidade própria. Assim, pode-se indicar que, principalmente no cenário brasileiro, as MR se aproximam de uma evolução dos portais de periódicos, em que os editores atuais de revistas pudessem atuar como se fossem editores de seção.

Nesse sentido, seria possível adaptar um portal de periódicos para tornar-se uma $M R$, ajustando as políticas editoriais, para que se tenha um processo mais dinâmico, advogando-se pela publicação em acesso aberto e contínua, sem a necessidade de organização em revistas e fascículos. Requer-se, no entanto, harmonização das políticas editoriais, na medida em que a dissolução das revistas disciplinares, impõe adaptações dos processos que atendam a todas as diferenças existentes.

É importante mencionar a manutenção da avaliação pelos pares, mesmo que implementem flexibilidades como defendidas pelo "deixe a comunidade decidir", do original "let the community decide", baseados no rigor do artigo. Entretanto, como relatam Spezi et al. (2018), na prática grande parte dos editores de MR utilizam a avaliação pelos pares de forma tradicional.

Quanto a sustentabilidade, pode-se implementar modelos voltados ao APC, quando aplicado, visto que o processo editorial é dispendioso e que o acesso aberto já apresenta modelos apropriados e validados. Entretanto uma ressalva deve ser feita quanto ao potencial aspecto 'caça níqueis', em que a MR cobra taxas a título de avaliação, mas na verdade não a realiza, publicando trabalhos na forma de 'pagar para publicar'.

Pontos relacionados à formatação dos artigos devem ser contemplados pela política, com indicação do formato Extensible Markup Language (XML), que facilita a extração automática de metadados e apresentação, visto poderem ser transformado facilmente para formatos, como o HTML. Regras de preservação e curadoria digital também devem ser contemplados, na medida em que é uma preocupação independente do tipo de revista.

Periódicos impressos levaram séculos para estabelecer-se e as MRs ainda requerem tempo para definir completamente as suas práticas, visto ainda ser novidade para muitos editores, autores e usuários. Discussões mais profundas, que estabeleçam modelos mais robustos, incluindo resultados de MRs estabelecidas. 


\section{REFERÊNCIAS}

BINFIELD, P. PLoS ONE and the rise of the Open Access MegaJournal. PLoS Biology, 2012. disponível em: <https://www.nii.ac.jp/sparc/en/event/2011/ pdf/20120229 doc3 binfield.pdf>. Acesso em: 20 abr. 2018.

BJÖRK, Bo-Christer; CATANI, P. Peer review in megajournals compared with traditional scholarly journals: Does it make a difference?. Learned publishing, v. 29, n. 1, p. 9-12, 2016. Disponível em: <https://onlinelibrary.wiley.com/doi/ full/10.1002/leap.1007>. Acesso em: 19 abr. 2018.

. Have the "mega-journals" reached the limits to growth?. PeerJ, v. 3, p. e981, 2015. Disponível em: <https://peerj.com/articles/981/>. Acesso em: 20 abr. 2018. e4357, 2018.

. Evolution of the scholarly mega-journal, 2006-2017. PeerJ, v. 6, p.

BURNS, C. S. Characteristics of a megajournal: a bibliometric case study. J Inf Sci Theory Pract. Korea Institute of Science and Technology Information, v. 3, p. 16-30, 2015.

DOMNINA, T. N. A megajournal as a new type of scientific publication. Scientific and Technical Information Processing, v. 43, n. 4, p. 241-250, 2016. Disponível em: <https://link.springer.com/article/10.3103\%2FS0147688216040079>. Acesso em: 22 abr. 2018.

FRANTSVÅG, J. E. The Mega-journals are coming!. ScieCom info. 2011. Disponível em: <https://munin.uit.no/handle/10037/3663 >. Acesso em: 19 abr. 2018.

HARNAD, S. et al. The access/impact problem and the green and gold roads to open access. 2004. Disponível em: <http://ost.openum.ca/files/sites/132/2017/06/access impact problem.pdf>. Acesso em: 9 maio 2018.

HARTGERINK, C. H. J.; VAN ZELST, M. "As-you-go" instead of" after-the-fact": A network approach to scholarly communication and evaluation. Peer] Preprints, 2018.

HURD, J. M. The transformation of scientific communication: A model for 2020. Journal of the Association for Information Science and Technology, $\mathrm{v}$. 51, n. 14, p. 1279-1283, 2000. Disponível em: <https://onlinelibrary.wiley.com/ doi/full/10.1002/1097-4571\%282000\%299999\%3A9999\%3C\%3A\%3AAID-ASI1044\%3E3.0.CO\%3B2-1>. Acesso em: 22 abr. 2018.

KRIEGESKORTE, N. Open evaluation: a vision for entirely transparent post-publication peer review and rating for science. Frontiers in computational neuroscience, v. 6, p. 79, 2012. 
LĂZĂROIU, G. Do mega-journals constitute the future of scholarly communication?. Educational Philosophy and Theory. 2017. Disponível em: <https://www.tandfonline.com/doi/full/10.1080/00131857.2017.1300022>. Acesso em: 19 abr. 2018.

\section{LÓPEZ-BORRULL, A. Megajournals, ¿̇hacia la posible fusión de revistas y} repositorios?. Anuario ThinkEPI 2014, v. 8, p. 203-206, 2014. Disponível em: <http://eprints.rclis.org/23318/>. Acesso em: 18 abr. 2018.

PACKER, A. L. The online modus operandi and the advancement of Brazilian nursing journals. Acta pulista de enfermagem, v. 25, n. 2, 2012. Dsponível em: < http:// www.scielo.br/scielo.php?pid=S0103-21002012000200001\&script=sci arttext\&tln$\mathrm{g}=\mathrm{es}>$. Acesso em: 16 abr. 2018.

PINFIELD, S.; WILLETT, P.; WAKELING, S. Open-access mega-journals. Cilip Update, 2017. Disponível em: <http://eprints.whiterose.ac.uk/115022/1/CILIP\%20 Update\%20for\%20PW.pdf>. Acesso em: 18 abr. 2018.

SPEZI, V. et al. Open-Access mega-journals: The future of scholarly communication or academic dumping ground? A review. Journal of Documentation, v. 73, n. 2, p. 263-283, 2017. Disponível em: <https://www.emeraldinsight.com/doi/ full/10.1108/JD-06-2016-0082>. Acesso em: 16 abr. 2018.

. "Let the community decide"? The vision and reality of soundness-only peer review in open-access mega-journals. Journal of Documentation, v. $74, n$. 1, p. 137-161, 2018.

SHOPOVSKI, J.; MAROLOV, D. Why Academics Choose to Publish in a Mega-Journal. Journal of Education and Learning, v. 6, n. 4, 2017. Disponível em: <https:// papers.ssrn.com/sol3/papers.cfm?abstract id=3032253 >. Acesso em: 15 abr. 2018.

TREINTA, F. T. et al. Metodologia de pesquisa bibliográfica com a utilização de método multicritério de apoio à decisão. Production, v. 24, n. 3, p. 508-520, 2014. Disponível em: <http://www.scielo.br/pdf/prod/2013nahead/aop prod0312.pdf>. Acesso em: 21 abr. 2018.

WAKELING, S. et al. Transitioning from a Conventional to a 'Mega'Journal: A Bibliometric Case Study of the Journal Medicine. Publications, v. 5, n. 2, p. 7, 2017. Disponível em: <http://www.mdpi.com/2304-6775/5/2/7/htm>. Acesso em: 20 abr. 2018. 